\title{
Bexl significantly contributes to the proliferation and invasiveness of malignant tumor cells
}

\author{
TAKEFUMI DOI $^{1}$, HIROYUKI OGAWA ${ }^{2}$, YUGO TANAKA ${ }^{1}$, YOSHITAKE HAYASHI $^{3}$ and YOSHIMASA MANIWA $^{1}$ \\ ${ }^{1}$ Division of Thoracic Surgery, Kobe University Graduate School of Medicine, Kobe, Hyogo 650-0017; \\ ${ }^{2}$ Department of Thoracic Surgery, Hyogo Cancer Center, Akashi, Hyogo 673-8558; \\ ${ }^{3}$ Division of Molecular Medicine and Medical Genetics, Department of Pathology, Kobe University \\ Graduate School of Medicine, Kobe, Hyogo 650-0017, Japan
}

Received January 7, 2020; Accepted July 15, 2020

DOI: $10.3892 / \mathrm{ol} .2020 .12226$

\begin{abstract}
Invasion has a significant role in cancer progression, including expansion to surrounding tissue and metastasis. Previously, we assessed the invasive ability of cancer cells using an easy-to-prepare double-layered collagen gel hemisphere (DL-CGH) method by which cancer cell invasion can be easily visualized. The present study examined multiple lung adenocarcinoma and malignant pleural mesothelioma (MPM) cell lines using the DL-CGH method and identified inherently invasive cell lines. Next, by comparing gene expression between invasive and non-invasive cells by cDNA microarray, the potential candidate gene brain-expressed $\mathrm{x}$-linked protein 1 (Bexl) was identified to be involved in cancer invasion, as it was highly expressed in the invasive cell lines. Downregulation of Bexl suppressed the invasion and proliferation of the invasive tumor cell lines. The findings of the present study suggested that Bexl may promote metastasis in vivo and could be a potential oncogene and molecular therapeutic target in lung adenocarcinoma and MPM.
\end{abstract}

\section{Introduction}

Lung cancer accounts for the majority of cancer-related deaths worldwide. Despite early detection with various methods, the majority of lung cancers are diagnosed at an advanced stage with metastasis to lymph nodes and/or distant organs. Therefore, lung cancer remains a poor prognostic tumor $(1,2)$.

Malignant pleural mesothelioma (MPM), which is a rare tumor compared with lung cancer, is a highly aggressive and poor prognostic tumor, mainly caused by exposure to asbestos (3). MPM mainly originates from mesothelial cells of

Correspondence to: Dr Yoshimasa Maniwa, Division of Thoracic Surgery, Kobe University Graduate School of Medicine, 7-5-2 Kusunoki-cho, Chuo-ku, Kobe, Hyogo 650-0017, Japan E-mail:maniwa@med.kobe-u.ac.jp

Key words: invasion, proliferation, Bexl, lung adenocarcinoma, malignant pleural mesothelioma the parietal pleura and is not likely to show metastasis to other organs (4). However, MPM shows locally aggressive invasion to the surrounding tissues, highlighting the malignancy of MPM (4-6).

Metastasis of cancer cells typically occurs through a series of steps: growth of cancer cells at the primary site, invasion to the extracellular matrix and into lymph and blood vessels, circulation in the vessels, extravasation out of the vessels, and finally, re-growth at distant organs $(7,8)$. Among these, invasion is one of the most important processes. The ability to invade reflects the malignancy of the cancer. In both lung cancer and MPM, invasion has a significant role in tumor progression and metastasis. Therefore, controlling invasion may lead to effective management of these tumors.

Previously, we established a simple 3-dimensional (3D) in vitro model of an invasion assay, which was referred to as the double-layered collagen gel hemisphere (DL-CGH) method (9). This method enables easy visualization of tumor cell invasion. Our previous study using the DL-CGH method demonstrated that the lung adenocarcinoma cell line A549 displayed no invasiveness on its own, but showed invasive migration in the presence of fibroblast cells (9). Previously, we also demonstrated the difference in cell invasiveness among MPM cell lines (10). These results indicated that the DL-CGH method could classify cancer cell lines into invasive or non-invasive, thereby allowing us to identify potential candidate gene(s) that are highly expressed in invasive cancer cell lines.

In the present study, the DL-CGH method was utilized and multiple cell lines were examined to identify potential candidate genes involved in cancer cell invasion. Cell invasion and proliferation were further evaluated in response to knocking down the candidate gene to determine its oncogenic potential.

\section{Materials and methods}

Cell lines. The human lung adenocarcinoma cell lines, A549 (bronchioloalveolar carcinoma of lung) and A110L, were purchased from the Riken Bioresource Center (A549, RCB0098; A110L, RCB2816). NCI-H28 (pleural effusion) and MSTO-211H (biphasic mesothelioma) were purchased from the American Type Culture Collection. These cells 


\section{Schematic of the structure of double-layered collagen gel hemispheres}

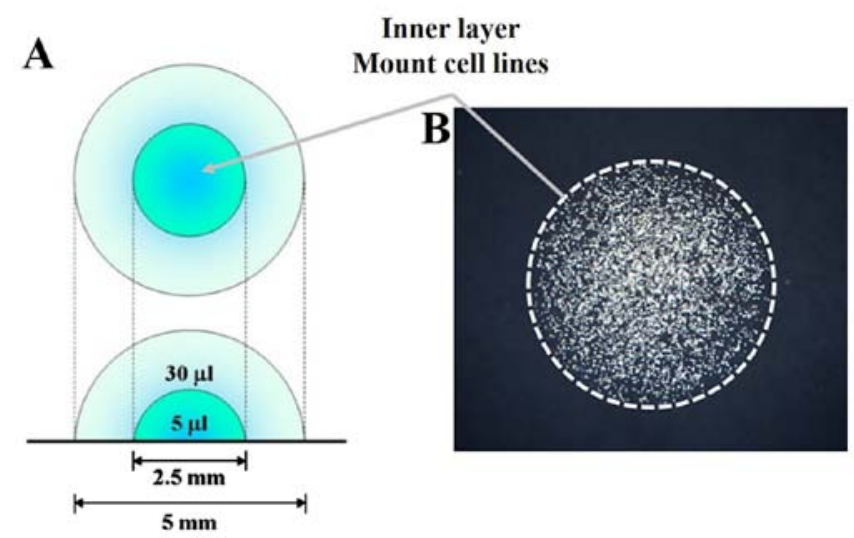

Figure 1. DL-CGH. (A) Schematic of the structure of DL-CGH. (B) Phase difference capture of DL-CGH immediately after mounting cell lines in the inner layer (day 0). DL-CGH, double-layered collagen gel hemispheres.

were subjected to mycoplasma testing prior to use in our experiments. The cells were cultured in RPMI-1640 medium (Sigma-Aldrich; Merck KGaA), supplemented with penicillin (100 U/ml), streptomycin (100 U/ml; GE Healthcare), and 10\% fetal bovine serum (FBS; Sigma-Aldrich) at $37^{\circ} \mathrm{C}$ in $5 \% \mathrm{CO}_{2}$.

Preparation of DL-CGH. Acid-soluble collagen I (Nitta Gelatin Inc.), 10-fold concentrated Ham's F-12 medium (Nitta Gelatin, NA Inc.), and reconstruction buffer $(2.2 \mathrm{~g}$ $\mathrm{NaHCO}_{3}+4.77 \mathrm{~g} \mathrm{HEPES}$ in $100 \mathrm{ml} 0.05 \mathrm{~N} \mathrm{NaOH}$ ) (Nitta Gelatin, NA Inc.) were mixed at a volume ratio of $8: 1: 1$ and then seeded with cultured cells at a density of $3.0 \times 10^{6}$ cells $/ \mathrm{ml}$. Five microliters of the mixture, containing $1.5 \times 10^{4}$ cells, were dispensed onto a plastic dish. Once the mixture had gelled, a second $30 \mu \mathrm{l}$ drop of collagen was placed exactly on the top of the first gel drop, encapsulating it completely (Fig. 1). The gel hemisphere was then submerged in medium and cultured.

Evaluation of lung adenocarcinoma and MPM cell invasion. Phase difference images were captured 0, 7, 10 and 14 days after the culture of cell lines with DL-CGH. Next, the cells were stained with neutral red solution (only taken in the viable cells) by reacting for $2 \mathrm{~h}$ with gentle shaking at $37^{\circ} \mathrm{C}$ in $5 \%$ $\mathrm{CO}_{2}$. The stained cell lines were subsequently fixed with $10 \%$ formalin neutral buffer solution (FUJIFILM Wako Pure Chemical Corp.) for $45 \mathrm{~min}$ at room temperature, washed with running water for $10 \mathrm{~min}$ and the gels were allowed to dry. The invasive activity of the cells was evaluated by measuring the expansion into the outer collagen layer. A Moticam 3 digital microscopy system (Shimadzu Rika Corp.) was used to capture phase difference images, particularly in evaluating the form of cell invasion. A BZ9000 fluorescence microscope (Keyence Corporation; magnification, $\mathrm{x} 50$ ) was used to evaluate the degree of cell invasion. For quantitative evaluation of viable cells with DL-CGH, Photoshop Elements 15 for Windows (Adobe Systems Inc.) was used. The red-stained areas in each image were selected manually. The 'histogram' selection in the pull-down menu then indicated the number of pixels with red-stained areas.
Total RNA isolation. Total RNA was isolated from the cell lines using TRIzol reagent (Thermo Fisher Scientific, Inc.) and purified using SV Total RNA Isolation System (Promega Corporation), according to the manufacturer's protocol. RNA samples were quantified by an ND-1000 spectrophotometer (NanoDrop Technologies; Thermo Fisher Scientific, Inc.) and the quality was confirmed using an Experion system (Bio-Rad Laboratories, Inc.).

Gene expression microarrays. The cRNA was amplified, labelled and hybridized to a 60K Agilent 60-mer oligomicroarray, according to the manufacturer's protocol. All hybridized microarray slides were scanned using an Agilent scanner (Agilent Technologies, Inc.). Relative hybridization intensities and background hybridization values were calculated using Agilent Feature Extraction Software (9.5.1.1; Agilent Technologies, Inc.). The array used was SurePrint G3 Human Gene Expression Microarray 8x60K v2 (model no. G4851A). A Low Input Quick Amp Labeling kit (model no. 5190-2305) was used to label reagent.

Data analysis and filter criteria. Raw signal intensities and flags for each probe were calculated from hybridization intensities (gProcessedSignal), and spot information (gIsSaturated), according to the following procedures recommended by Agilent Flag criteria on GeneSpring Software: Absent (A): 'Feature is not positive and significant' and 'Feature is not above background'; Marginal (M): 'Feature is not Uniform' 'Feature is Saturated' and 'Feature is a population outlier'; Present (P): others.

The raw signal intensities of two samples were $\log _{2}$-transformed and normalized by quantile algorithm with 'preprocessCore' library package (11) on Bioconductor software (12).

Probes that call at least one sample ' $\mathrm{P}$ ' flag, excluding lincRNA probes, were selected. To identify upregulated or downregulated genes, Z-scores (13) and ratios (non-log scaled fold-change) were calculated from the normalized signal intensities of each probe for comparison between control and experiment samples. Next, criteria were established for 

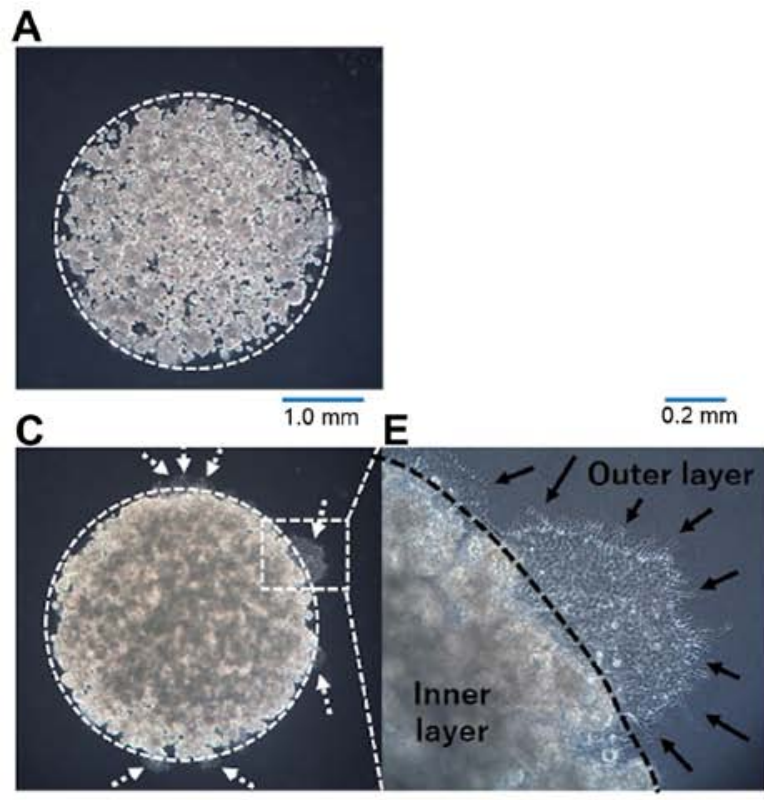

B
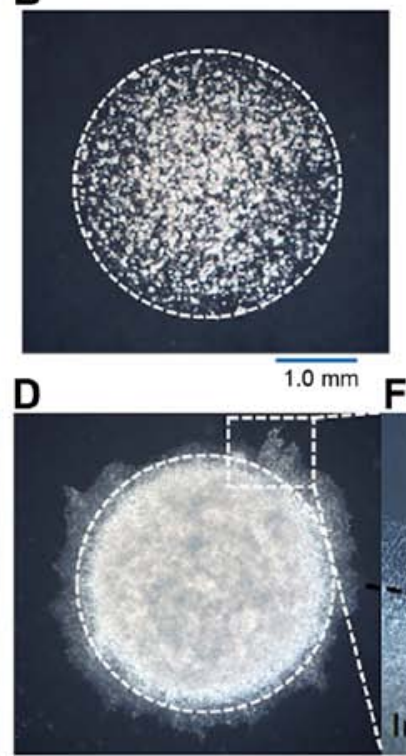

\section{$\mathrm{F}$}

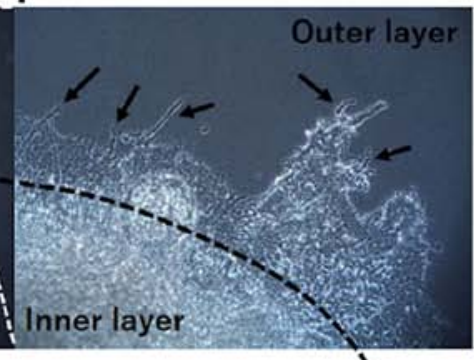

Figure 2. Result of DL-CGH using lung adenocarcinoma and MPM cell lines. (A) A549, (B) NCI-H28, (C) A110L and (D) MSTO-211H cells. Lung adenocarcinoma cell lines were compared at day 14, and those of MPM were compared at day 10. The difference in capture day depended on the speed of cell proliferation in lung adenocarcinoma and MPM. A549 and NCI-H28 cells exhibited almost no invasion to the outer layer. A110L and MSTO-211H cells exhibited a high tendency to invade to the outer layer (dashed arrow). Enlarged images of boundary between the inner layer and the outer layer in the DL-CGH (E, A110L; F, MSTO-211H). A110L and MSTO-211H exhibit a dendriform shape (arrow). DL-CGH, double-layered collagen gel hemispheres; MPM, malignant pleural mesothelioma.

regulated genes: (upregulated genes) Z-score $\geq 2.0$ and ratio $\geq 1.5$-fold, (downregulated genes) Z-score $\leq-2.0$ and ratio $\leq 0.66$.

Western blotting. Cultured cells were lysed with $100 \mu 1$ Laemmli sample buffer (Cosmo Bio Co., Ltd.), and $10 \mu \mathrm{l}$ of each sample lysate was run on SDS-PAGE using Any $\mathrm{kD}^{\mathrm{TM}}$ Mini-PROTEAN ${ }^{\circledR}$ TGX $^{\mathrm{TM}}$ Precast Gels (Bio-Rad Laboratories, Inc.). Next, the separated bands were transferred to nitrocellulose membranes (GE Healthcare). After washing the membranes with phosphate buffered saline-Tween-20 (PBS-T, including 0.05\% Tween-20), they were blocked for $60 \mathrm{~min}$ at room temperature, with $2 \%$ ECL Prime Blocking Reagent (GE Healthcare), diluted by PBS-T. Following 3 rinses with PBS-T, membranes were incubated $(60 \mathrm{~min}$, $15-25^{\circ} \mathrm{C}$ ) with the primary rabbit polyclonal antibody to brain-expressed x-linked protein 1 (Bexl, $15 \mathrm{kDa}$; cat. no. ab107215; Abcam), which was diluted 1:500 with 5\% bovine serum albumin/PBS-T. Following washing with PBS-T, membranes were incubated (30 min, room temperature) with the secondary peroxidase-labeled sheep anti-rabbit Ig whole antibody (cat. no. NA934; GE Healthcare), which was diluted 1:5,000 with PBS-T. Membranes were then washed with PBS-T and visualized using a luminoimage analyzer Amersham Imager 600 (Fuji film, Inc.) treated with a chemiluminescent detection kit (GE Healthcare). When the expression of Bexl protein was upregulated, the $15 \mathrm{kDa}$ band was deeply stained on western blotting.

For the control assay, western blotting was performed using the same membranes. The primary antibody was directed against $\alpha$-tubulin mouse monoclonal antibody (1:1,000; cat. no. 017-25031; Wako Pure Chemical Industries, Ltd.), and the secondary antibody was peroxidase-labelled sheep anti-mouse Ig whole antibody (GE Healthcare).

Real time-polymerase chain reaction (RT-PCR) analysis in cell lines. Total RNA was extracted as mentioned earlier. Using $1 \mu \mathrm{g}$ of the collected total RNA, ReverTra Ace ${ }^{\circledR}$ qPCR RT Master Mix with gDNA Remover (Toyobo Life Science) was used to decompose chromosomal DNA by DNase treatment, according to the manufacturer's protocol, and then cDNA was synthesized by reverse transcription. Gene expression analysis of $\beta$-actin (assay ID: Hs01060665_g1, housekeeping gene) and BEX1 (assay ID: Hs00218464_m1) were performed by quantitative PCR with Taqman ${ }^{\circledR}$ Gene Expression Assays (Applied Biosystems; Thermo Fisher Scientific, Inc.), according to the manufacturer's protocol.

RNA interference in lung adenocarcinoma and MPM cell lines. RNA interference (RNAi) was performed with commercially available siRNAs for Bexl (\#s31681 and \#s226875; Invitrogen) and a non-silencing control siRNA (Invitrogen), according to the manufacturer's protocol. In the present study, reverse transfection of RNAi was performed. Briefly, 15 pmol (7.5 pmol \#s31681 and 7.5 pmol \#s226875) transfection reagent was suspended in $2.5 \mu \mathrm{l}$ Lipofectamine RNAiMAX (Invitrogen) and $500 \mu \mathrm{l}$ Opti-MEM (Invitrogen), finally containing $5 \mu \mathrm{M}$ siRNA. Following a 5-min incubation at room temperature, the complexes were prepared inside a $35-\mathrm{mm}$ round culture dish, after which the cell lines were incubated at $37^{\circ} \mathrm{C}$ in $5 \%$ $\mathrm{CO}_{2}$, with $2.5 \mathrm{ml}$ culture medium containing $10 \% \mathrm{FBS}$ and the aforementioned antibiotics and cultured for $48 \mathrm{~h}$. The cell lines were cultured for $48 \mathrm{~h}$ (at $37^{\circ} \mathrm{C}, 5 \% \mathrm{CO}_{2}$ ) until they reached $80 \%$ confluence; the final concentration of the siRNA was 

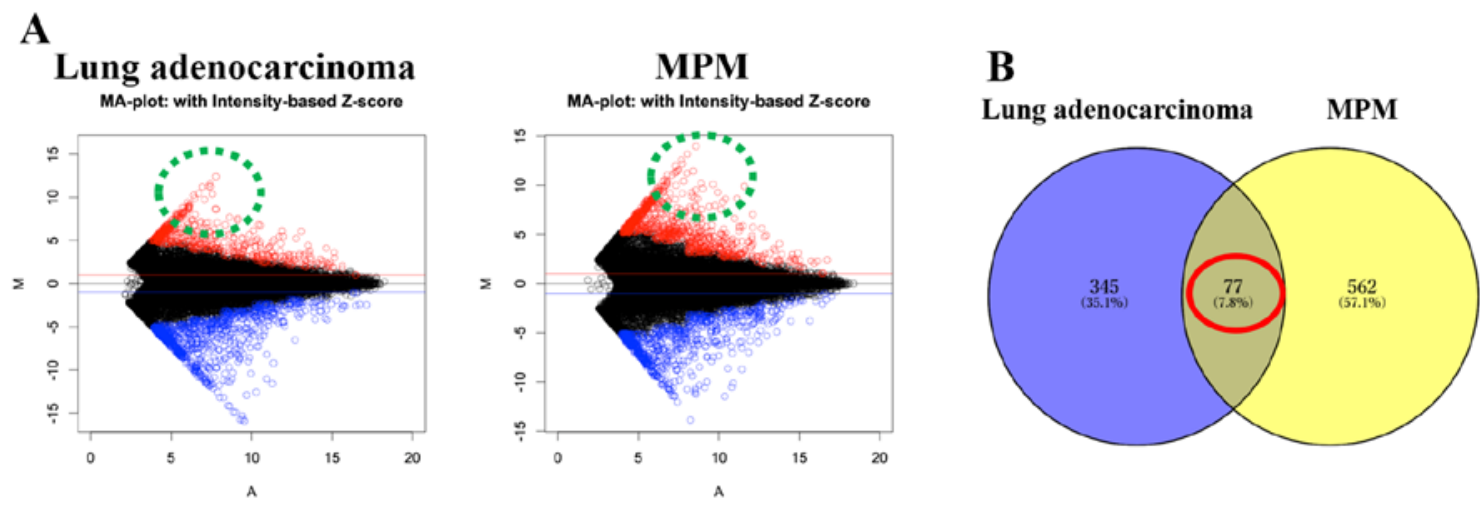

Lung adenocarcinoma : A110L vs A549

MPM : MSTO-211H vs NCI-H28

Figure 3. Differential gene expression in lung adenocarcinoma and MPM cell lines. (A) MA-plot. Lung adenocarcinoma cell lines (A110L vs. A549); MPM cell lines (MSTO-211H vs. NCI-H28). (B) Venn diagram. MPM, malignant pleural mesothelioma.

$5 \mathrm{nM}$. After $48 \mathrm{~h}$, these cells were used for DL-CGH and cell proliferation assays. Cell density was calculated to prepare for encapsulation of the cells in DL-CGH.

Assessment of proliferation in lung adenocarcinoma and MPM cell lines. Cell lines were cultured on normal tissue culture plates $(60-\mathrm{mm}$ round dish with $4 \mathrm{ml}$ medium, containing $10 \%$ FBS and the aforementioned antibiotics). These plates contained $20 \times 10^{4}$ cells at day 0 , and the cells were cultured and the cell population counted every $24 \mathrm{~h}$ from day 0 to day 3 (72 h).

Statistical analysis. Multiple comparisons of viable cells were assessed by counting the pixel numbers of red-stained areas using Tukey HSD test. A repeated measures analysis of variance was used for the analysis of the comparison of cell proliferative activity in A110L and MSTO-211H cells. All tests were performed using JMP ver. 12.0 statistical software (SAS Institute, Inc.). $\mathrm{P}<0.05$ was considered to indicate a statistically significant difference.

\section{Results}

Selection of lung adenocarcinoma and MPM cell lines for invasion. In our previous study assessing invasion in lung adenocarcinoma cell lines using the DL-CGH methodology, A549 cells showed no invasion into the outer layer (9). It was also demonstrated that NCI-H28 cells had no invasiveness, while MSTO-211H cells exhibited a high tendency to invade the outer layer (10). A previous study from another group revealed that the $\mathrm{A} 110 \mathrm{~L}$ cell line presented a high invasion ratio (14), suggesting that $\mathrm{A} 110 \mathrm{~L}$ would display invasiveness in the DL-CGH assay. In the present study, DL-CGH was performed using these cell lines and the invasiveness of the lung adenocarcinoma and MPM cell lines was confirmed. A549 and NCI-H28 cells exhibited almost no invasion into the outer layer (Fig. 2A and B), while A110L and MSTO-211H cells demonstrated strong invasion to the outer layer (Fig. 2C and D).
Pattern of invasion observed by DL-CGH. Invasive cells (A110L and MSTO-211H) was observed at the boundary between the inner layer and the outer layer in the DL-CGH. The invasive cells in these cell lines spread with dendriform extension to the outer layer (Fig. 2E and F). A549 cells, which had almost no invasive ability, slightly extended to the outer layer. However, A549 cells did not present with a dendritic shape, and it was hypothesized that the increased A549 cells were merely pushed out to the outer layers and did not consider this to be true invasion.

cDNA microarray: Invasive cells vs. non-invasive cells. It was assumed that the genes involved in cancer cell invasion may be common even if the cancer types are different. Therefore, gene expression was compared between invasive and non-invasive cells of lung adenocarcinoma and MPM. From the results of the cDNA microarray, gene expression was compared between the invasive and non-invasive cells. Next, the focus was on the genes that were more highly expressed in invasive cells than in non-invasive cells of lung adenocarcinoma and MPM, respectively (Fig. 3A). The genes were further sorted, showing more than 100-fold expression in invasive cells than in non-invasive cells in both lung adenocarcinoma and MPM (Fig. 3B; Table I). Among these genes, the brain expressed X-linked 1 (Bexl) gene was selected as a candidate gene that was most expressed in invasive cells (A110L and MSTO-211H cell lines). Upon comparison of the $\mathrm{Z}$ score ratio, the expression of Bexl in A110L cells was 2,354 times that in A549 cells (lung adenocarcinoma) and that in MSTO-211H was 3,951 times that in NCI-H28 (MPM), respectively (Table I).

Effect of inhibition of Bexl in A110L and MSTO-211H cells on invasion. Western blotting was performed to confirm the expression of Bexl in the invasive and non-invasive cell lines. Western blotting revealed strong expression of Bexl in A110L and MSTO-211H cells (Fig. 4A), along with corresponding high gene expression of Bexl. Additionally, RT-PCR revealed a higher expression level of Bexl in A110L and MSTO-211H cells than in A549 and NCI-H28 cells (Table II). Next, RNAi 


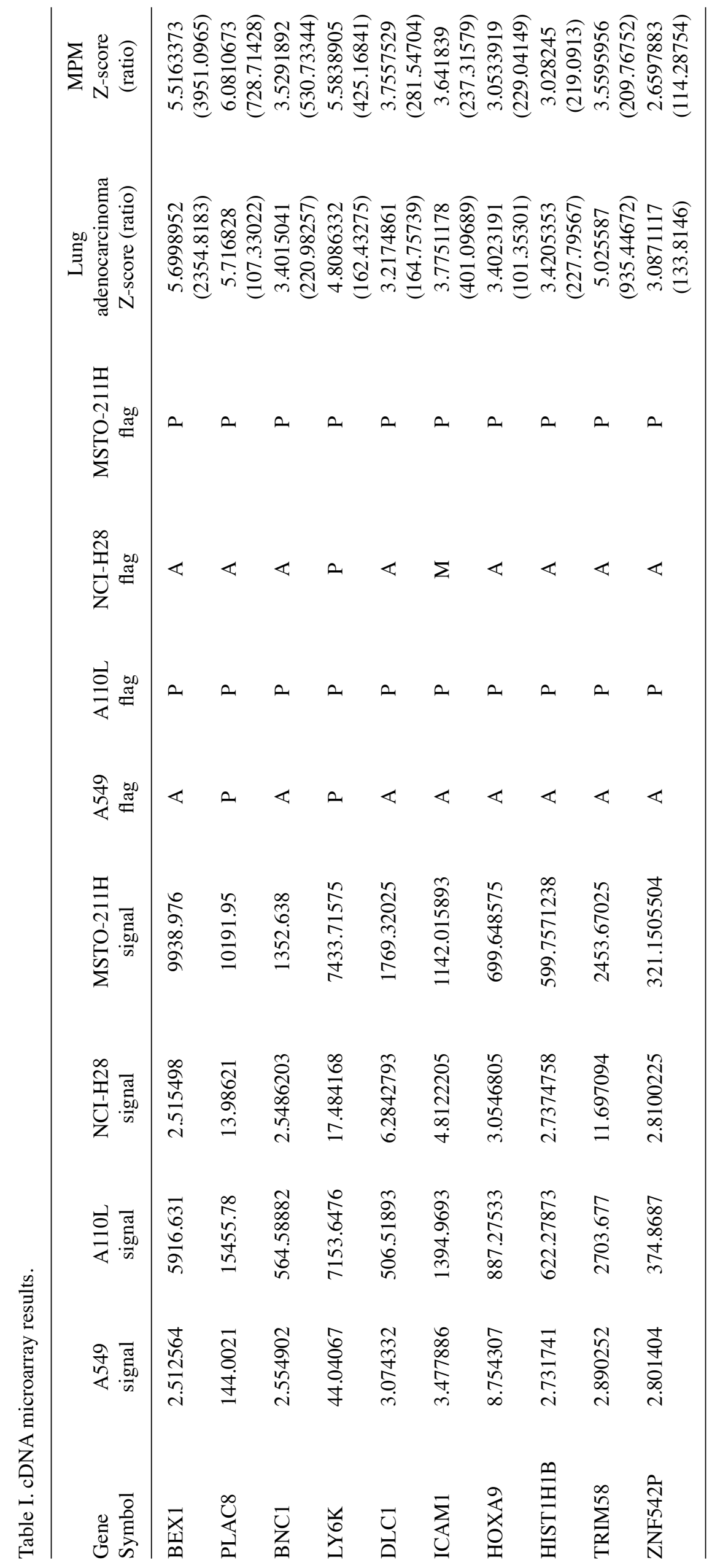


A
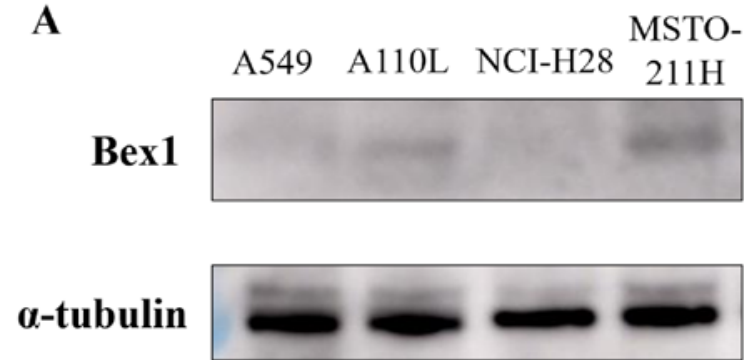

B

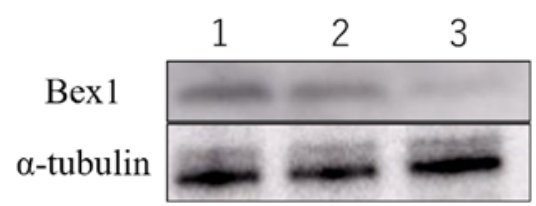

1. Non-treated

2. Control non-silencing si RNA

3. Bex1-siRNA
C

\section{MSTO-211H}

123

$\operatorname{Bex} 1$

$\alpha$-tubulin

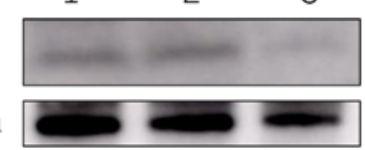

Figure 4.Bexl protein expression in lung adenocarcinoma and MPM cell lines. (A) Expression of Bexl was higher in invasive cell lines (A110L and MSTO-211H) than in non-invasive cell lines (A549 and NCI-H28). Bexl-knockdown was confirmed by western blotting in (B) A110L and (C) MSTO-211H cells in the background of the following treatments: Control-treated (only Lipofectamine, 1), control non-silencing siRNA-treated (2), and Bexl-siRNA-treated (3). MPM, malignant pleural mesothelioma; siRNA, small interfering RNA; Bexl, brain-expressed X-linked protein 1.

Table II. Relative Bexl expression.

\begin{tabular}{cccc}
\hline A549 & A110L & NCI-H28 & MSTO-211H \\
\hline 0.00306 & 0.507 & ND $(<0.000228)$ & 1.506 \\
\hline
\end{tabular}

Since the Ct value could not be obtained with NCI-H28, the Ct value was calculated as $<40$. ND, not detected.

methodology was used to knock down the expression of Bexl in A110L and MSTO-211H cells (Fig. 4B and C) and the association between Bexl expression and cell invasion was observed using the DL-CGH. A110L and MSTO-211H cells with reduced Bexl expression showed almost no invasion to the outer layer according to DL-CGH (Figs. 5A and 6A). Furthermore, the cells around the boundary of the inner layer and the outer layer did not present with a dendriform shape and exhibited almost no tendency to invade the outer layer (Figs. 5B and 6B). In addition, the comparison of DL-CGH stained live cells with neutral red at day 0 and day 14 (A110L) or day $10($ MSTO-211H) showed that siRNA-induced Bexl-knockdown led to a substantial reduction in live cells, suggesting that Bexl promotes viability (Figs. $5 \mathrm{C}$ and D and $6 \mathrm{C}$ and $\mathrm{D})$.

Effect of RNAi-mediated knockdown of Bexl on cell proliferation. Notably, suppressing Bexl expression not only eliminated the invasive ability of the cells, but also decreased the cell density in the inner layer of DL-CGH. Using A110L and MSTO-211H cells with Bexl knocked down, the proliferative effects of Bexl were also assessed. For assessment of cell proliferation using A110L and MSTO-211H cells, normal cell lines were compared with control non-silencing siRNA and Bexl RNAi in each cell line. As a result of Bexl-knockdown, A110L and MSTO-211H exhibited significantly reduced cell proliferation (Fig. 7).

\section{Discussion}

The present study used the DL-CGH method to visually classify the lung adenocarcinoma and MPM cell lines as invasive or non-invasive. Additionally, it was assumed that genes involved in cancer cell invasion may be common even if the cancer types were different, and potential genes were identified that were overexpressed in the invasive cell lines of lung adenocarcinoma and MPM, respectively, to a greater extent than in the non-invasive cell lines. Among these, Bexl was examined as a candidate gene that was highly expressed in invasive cell lines.

Previous studies have made use of various methods for in vitro 3D models of invasion with collagen gel, including our method (9,15-17). Among them, our DL-CGH method has an advantage in terms of simplicity and ease of preparation, only involving two collagen gel drops with cell lines enclosing the inner collagen gel drop (9). Furthermore, DL-CGH may aid in visually categorizing cell lines into invasive or non-invasive, as the dynamics of cell lines at the boundary between the inner and outer layer of the DL-CGH can be observed. This visualization of cell invasion is very useful, not only for observing the mechanics of cancer cell invasion, but also as a screening technique for cell invasiveness.

The human brain expressed X-linked (Bex) family proteins consist of five proteins (Bexl-5) and are known as material proteins in neuronal development (18). For example, Bexl is involved in axon regeneration (19). In addition to this function, Bexl interacts with the p75 neurotrophin receptor (NTR) and 

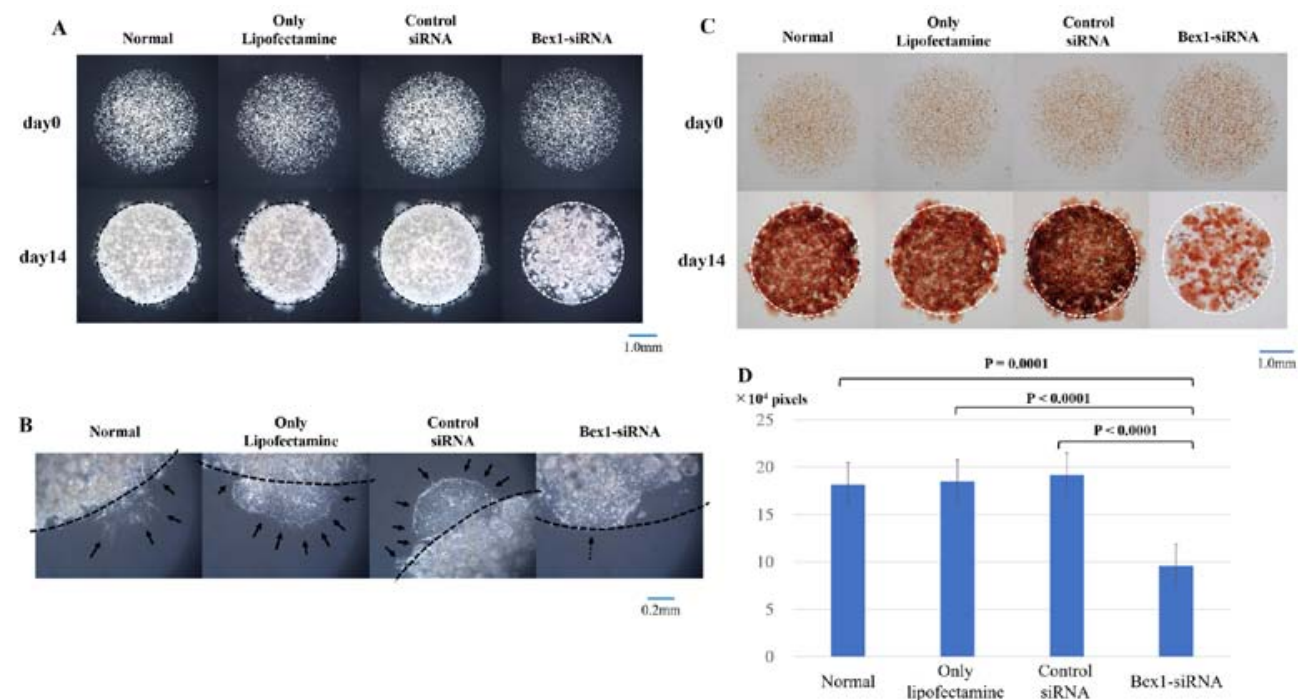

Figure 5. Invasion is suppressed in A110L cells with reduced Bexl expression. The degree of invasion was compared in A110L cells using the DL-CGH assay and the following groups were compared: non-treated, control-treated (only Lipofectamine), control non-silencing siRNA-treated, and Bexl-siRNA-treated. (A) The comparison of DL-CGH in phase difference capture at day 0 and day 14. Knocking down of Bexl induced strong suppression of cell invasion to the outer layer with DL-CGH. (B) Enlarged images of boundary between the inner layer and the outer layer. A110L with expression of Bexl showed dendriform extension (arrow), while A110L with Bexl knocked down did not present with a dendriform shape (dashed arrow). (C) The comparison using DL-CGH of living cells stained with neutral red at day 0 and day 14. Bexl RNAi induced prominent cell reduction. (D) The histogram showed the mean and standard deviation of numbers of red-stained pixels of each DL-CGH sample at day 14. The pixel numbers of red-stained areas were 181,748 in normal (non-treated), 184,928 in control-treated (only Lipofectamine), 191,934 in control non-silencing siRNA-treated, and 95,914 in Bexl-siRNA-treated. The pixel numbers of Bexl RNAi were significantly decreased. Bexl, brain-expressed X-linked protein 1; siRNA, small interfering RNA; DL-CGH, double-layered collagen gel hemispheres.
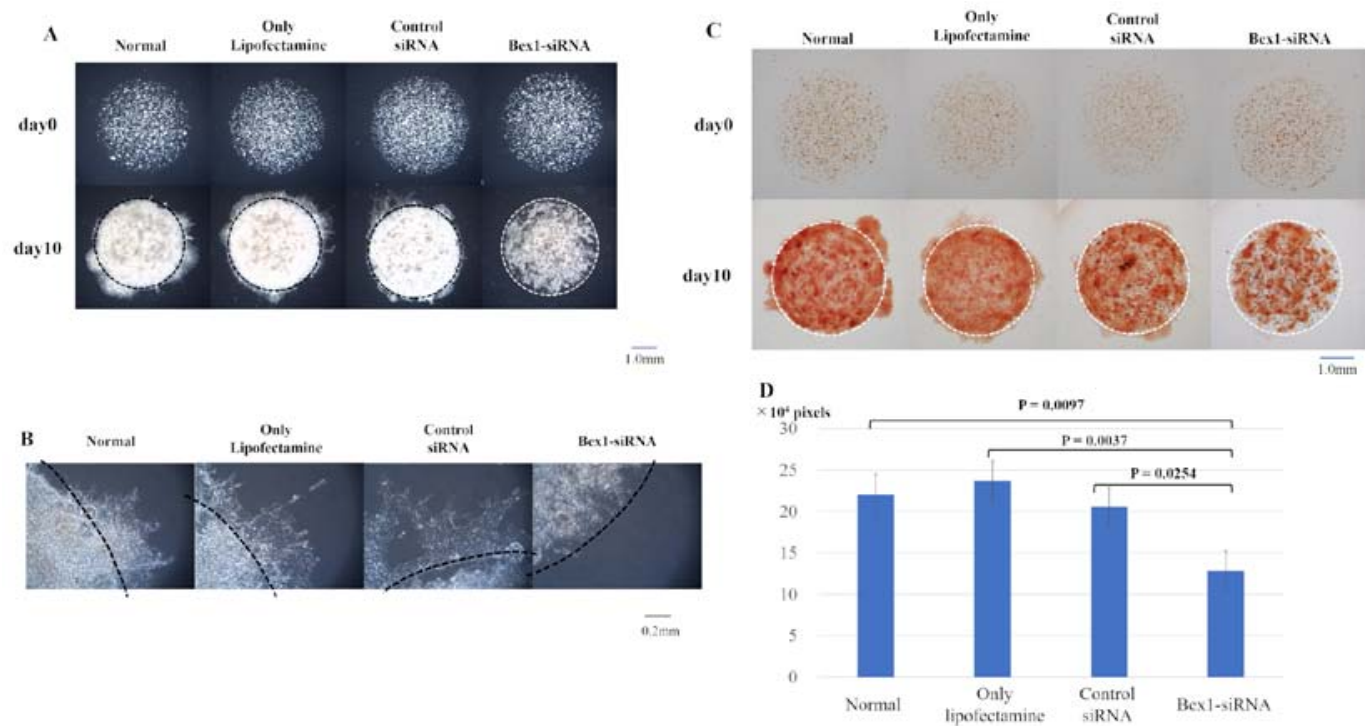

Figure 6. Invasion is suppressed in MSTO-211H cells with reduced Bexl expression. The degree of invasion in MSTO-211H cells was compared using the DL-CGH assay and comparisons were made between the following cells: Non-treated, control-treated (only Lipofectamine), control non-silencing siRNA-treated, and Bexl-siRNA-treated. (A) Comparison using DL-CGH of phase difference capture at day 0 and day 14. (B) Enlarged images of boundary between the inner layer and the outer layer. MSTO-211H with Bexl knocked down showed almost no cells with dendriform shape. (C) Comparison using DL-CGH of living cells stained with neutral red at day 0 and day 14. (D) Histogram showed the mean and standard deviation of numbers of red-stained pixels of each DL-CGH sample at day 10. The pixel numbers of red-stained areas were 220,502 in normal (non-treated), 236,713 in control-treated (only Lipofectamine), 205,433 in control non-silencing siRNA-treated, and 128,262 in Bexl-siRNA-treated. The pixel numbers of Bexl RNAi were also significantly decreased. Bexl, brain-expressed X-linked protein 1; siRNA, small interfering RNA; DL-CGH, double-layered collagen gel hemispheres.

contributes toward regulation of the cell cycle (20). Previous studies have demonstrated that Bex family proteins are associated with several human cancer types. In breast cancer, overexpression of Bexl and Bex2 led to inhibition of tumor cell apoptosis $(21,22)$ and Bexl acts as a resistance marker for chemotherapy (23). Bexl is also known to be involved in the tumorigenesis of neuroendocrine-specific tumors (24). However, in malignant glioma, the expression of Bexl and Bex2 was suppressed in human glioma cell lines and primary patient samples (25). In contrast to our findings, overexpression of Bexl in intracranial ependymoma in children significantly suppressed cell proliferation and colony formation (26). 

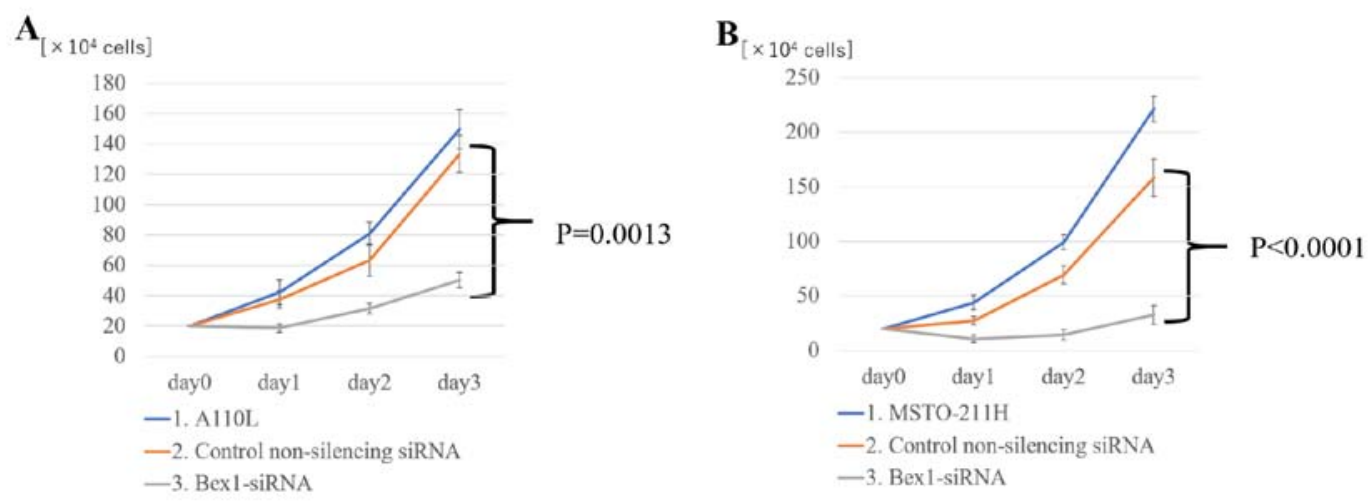

Figure 7. Bexl promotes the proliferation of A110L and MSTO-211H cells. In (A) A110L and (B) MSTO-211H cells, proliferation was strongly suppressed in the cells with Bexl knocked down (n=5 samples for each group). (1) control-treated (only Lipofectamine), (2) control non-silencing siRNA-treated, and (3) Bexl-siRNA-treated. Bexl, brain-expressed X-linked protein 1; siRNA, small interfering RNA.

However, loss of Bexl expression in acute myeloid leukemia resulted in decreased cell proliferation, colony and tumor formation, and increased apoptosis, suggesting that Bexl is an oncogene in this model (27). In other studies, downregulation of Bexl contributed toward imatinib resistance and inhibition of apoptosis in chronic myeloid leukemia $(28,29)$. Despite numerous studies on the function of Bexl in various cancer types, there has been no investigation into its role in lung adenocarcinoma and MPM, making this study the first to analyze the association between Bexl in lung adenocarcinoma and MPM.

The present study demonstrated that Bexl serves an important role in cell proliferation and the invasive activity of lung adenocarcinoma and MPM cells. Downregulation of Bexl decreased the proliferation and invasion of these cancer cells, indicating that Bexl is a candidate oncogene in lung adenocarcinoma and MPM. Furthermore, we hypothesized that Bexl is involved in cell proliferation and cell death. In the evaluation of Fig. 7 at day 1 (one day after administration of Bexl-siRNA), it was considered that cell death was induced to a certain degree because the numbers of A110L and MSTO-211H cells were slightly decreased. However, subsequent changes in Fig. 7 (day 2 and day 3) and the result of quantification of cell viability by staining with neutral red revealed that the speed was decreasing, but the cell proliferation itself was progressing. These results indicated that Bexl may be more effective in suppressing cell proliferation than in inducing cell death.

A previous study revealed that the presence of fibroblasts was important for cancer cell invasion $(30,31)$ and that cancer cells with no inherent invasive capacity slipped along the gaps of extracellular matrix created by the fibroblasts $(9,32)$. However, certain cancer cells present inherent invasive ability. In invasive single-cell migration, cancer cells change their form to spindle-like shapes with pseudopod protrusion (33-35). In our previous study (10) and in the present study, the DL-CGH results demonstrated that certain cell lines of both MPM and lung adenocarcinoma had an inherent ability of invasion, in the absence of fibroblasts. Specifically, we observed A110L and MSTO-211H cells independently spread with dendriform extension to the outer layer. In particular, A110L cells (lung adenocarcinoma) changed their form into spindle shapes at the site of invasion in the DL-CGH. The dendritic formation of these cancer cells was suppressed by knockdown of Bexl, following which they could not spread into the outer layer at the DL-CGH (Figs. 5B and 6B). This result indicated that Bexl expression is likely involved, not only in cell proliferation, but also in the ability of invasion by dendritic formation. Furthermore, suppression of Bexl in cancer cells that overexpress Bexl may lead to control of local invasiveness and improved cancer treatment.

The main purpose of the present study was to report urgently that a candidate gene involved in cell invasion and proliferation could be sorted by comparing several cell lines applying the DL-CGH method, and from this result, that Bexl could be listed as a candidate gene involved in cell invasion and proliferation. Therefore, the Bexl gene was successfully knocked down, which was performed relatively quickly. In a future study, we are planning an assessment of Bexl overexpression in non-invasive cells through an in vivo study and a study using clinical samples.

In conclusion, the results of the present study demonstrated that the overexpression of Bexl promotes tumor proliferation and invasion to the surrounding tissue, and thus identifies Bexl as a candidate oncogene in lung adenocarcinoma and MPM. Furthermore, knocking down of Bexl strongly inhibits the ability of cells to invade and proliferate in vitro, indicating that Bexl may be a molecular target for the treatment of lung adenocarcinoma and MPM.

\section{Acknowledgements}

Not applicable.

\section{Funding}

The present study was supported by the Japan Society for the Promotion of Science (JSPS) KAKENHI, Grant-in-Aid for Scientific Research (B) (grant no. 17H04297).

\section{Availability of data and materials}

All data generated and/or analyzed during this study are included in this published article. 


\section{Authors' contributions}

TD, YH and YM developed the concept, designed the experiments, and drafted the initial manuscript. TD performed the experiments and analyzed the data. HO, YT and YM helped analyze the cDNA microarray data and identify the candidate gene. HO and YT helped perform western blotting. All authors read and approved the final manuscript.

\section{Ethics approval and consent to participate}

Not applicable.

\section{Patient consent for publication}

Not applicable.

\section{Competing interests}

The authors declare that they have no competing interests.

\section{References}

1. Herbst RS, Heymach JV and Lippman SM: Lung cancer. N Engl J Med 359: 1367-1380, 2008.

2. Cheng TY, Cramb SM, Baade PD, Youlden DR, Nwogu C and Reid ME: The international epidemiology of lung cancer: Latest trends, disparities, and tumor characteristics. J Thorac Oncol 11: $1653-1671,2016$.

3. Robinson BW, Musk AW and Lake RA: Malignant mesothelioma. Lancet 366: 397-408, 2005.

4. Pistolesi M and Rusthoven J: Malignant pleural mesothelioma: Update, current management, and newer therapeutic strategies. Chest 126: 1318-1329, 2004.

5. Antman KH: Natural history and epidemiology of malignant mesothelioma. Chest 103: 373S-376S, 1993.

6. Zhong J, Gencay MM, Bubendorf L, Burgess JK, Parson H, Robinson BW, Tamm M, Black JL and Roth M: ERK1/2 and p38 MAP kinase control MMP-2, MT1-MMP, and TIMP action and affect cell migration: A comparison between mesothelioma and mesothelial cells. J Cell Physiol 207: 540-552, 2006.

7. Chambers AF, Groom AC and MacDonald IC: Dissemination and growth of cancer cells in metastatic sites. Nat Rev Cancer 2 563-572, 2002

8. Sahai E: Illuminating the metastatic process. Nat Rev Cancer 7: 737-749, 2007.

9. Takata M, Maniwa Y, Doi T, Tanaka Y, Okada K, Nishio W, Ohbayashi C, Yoshimura M, Hayashi Y and Okita Y: Double-layered collagen gel hemisphere for cell invasion assay: Successful visualization and quantification of cell invasion activity. Cell Commun Adhes 14: 157-167, 2007.

10. Doi T, Maniwa Y, Tanaka Y, Tane S, Hashimoto S, Ohno Y, Nishio W, Nishimura Y, Ohbayashi C, Okita Y, et al: MT1-MMP plays an important role in an invasive activity of malignant pleural mesothelioma cell. Exp Mol Pathol 90: 91-96, 2011.

11. Bolstad BM, Irizarry RA, Astrand M and Speed TP: A comparison of normalization methods for high density oligonucleotide array data based on variance and bias. Bioinformatics 19: 185-193, 2003.

12. Gentleman RC, Carey VJ, Bates DM, Bolstad B, Dettling M, Dudoit S, Ellis B, Gautier L, Ge Y, Gentry J, et al: Bioconductor: Open software development for computational biology and bioinformatics. Genome Biol 5: R80, 2004.

13. Quackenbush J: Microarray data normalization and transformation. Nat Genet 32: 496-501, 2002.

14. Izumi H, Takahashi M, Uramoto H, Nakayama Y, Oyama $T$, Wang KY, Sasaguri Y, Nishizawa S and Kohno K: Monocarboxylate transporters 1 and 4 are involved in the invasion activity of human lung cancer cells. Cancer Sci 102 : 1007-1013, 2011.

15. Albini A, Iwamoto Y, Kleinman HK, Martin GR, Aaronson SA, Kozlowski JM and McEwan RN: A rapid in vitro assay for quantitating the invasive potential of tumor cells. Cancer Res 47 3239-3245, 1987
16. Nyström ML, Thomas GJ, Stone M, Mackenzie IC, Hart IR and Marshall JF: Development of a quantitative method to analyse tumour cell invasion in organotypic culture. J Pathol 205: 468-475, 2005.

17. Duong HS, Le AD, Zhang Q and Messadi DV: A novel 3-dimensional culture system as an in vitro model for studying oral cancer cell invasion. Int J Exp Pathol 86: 365-374, 2005.

18. Kazi JU, Kabir NN and Rönnstrand L: Brain-Expressed X-linked (BEX) proteins in human cancers. Biochim Biophys Acta 1856: 226-233, 2015.

19. Khazaei MR, Halfter H, Karimzadeh F, Koo JH, Margolis FL and Young P: Bex1 is involved in the regeneration of axons after injury. J Neurochem 115: 910-920, 2010.

20. Vilar M, Murillo-Carretero M, Mira H, Magnusson K, Besset V and Ibáñez CF: Bex1, a novel interactor of the p75 neurotrophin receptor, links neurotrophin signaling to the cell cycle. EMBO J 25: 1219-1230, 2006

21. Naderi A, Teschendorff AE, Beigel J, Cariati M, Ellis IO, Brenton JD and Caldas C: BEX2 is overexpressed in a subset of primary breast cancers and mediates nerve growth factor/nuclear factor-kappaB inhibition of apoptosis in breast cancer cell lines. Cancer Res 67: 6725-6736, 2007.

22. Naderi A, Liu J and Bennett IC: BEX2 regulates mitochondrial apoptosis and G1 cell cycle in breast cancer. Int J Cancer 126: 1596-1610, 2010

23. de Ronde JJ, Lips EH, Mulder L, Vincent AD, Wesseling J, Nieuwland M, Kerkhoven R, Vrancken Peeters MJ, Sonke GS, Rodenhuis S, et al: SERPINA6, BEX1, AGTR1, SLC26A3, and LAPTM4B are markers of resistance to neoadjuvant chemotherapy in HER2-negative breast cancer. Breast Cancer Res Treat 137: 213-223, 2013

24. Hofsli E, Wheeler TE, Langaas M, Laegreid A and Thommesen L: Identification of novel neuroendocrine-specific tumour genes. $\mathrm{Br}$ J Cancer 99: 1330-1339, 2008.

25. Foltz G, Ryu GY, Yoon JG, Nelson T, Fahey J, Frakes A, Lee H, Field L, Zander K, Sibenaller Z, et al: Genome-wide analysis of epigenetic silencing identifies BEX1 and BEX2 as candidate tumor suppressor genes in malignant glioma. Cancer Res 66: 6665-6674, 2006.

26. Karakoula K, Jacques TS, Phipps KP, Harkness W, Thompson D, Harding BN, Darling JL and Warr TJ: Epigenetic genome-wide analysis identifies BEX1 as a candidate tumour suppressor gene in paediatric intracranial ependymoma. Cancer Lett 346: 34-44, 2014.

27. Lindblad O, Li T, Su X, Sun J, Kabir NN, Levander F, Zhao H, Lu G, Rönnstrand L and Kazi JU: BEX1 acts as a tumor suppressor in acute myeloid leukemia. Oncotarget 6: 21395-21405, 2015.

28. Ding K, Su Y, Pang L, Lu Q, Wang Z, Zhang S, Zheng S, Mao J and Zhu Y: Inhibition of apoptosis by downregulation of hBex1, a novel mechanism, contributes to the chemoresistance of $\mathrm{Bcr} / \mathrm{Abl}^{+}$ leukemic cells. Carcinogenesis 30: 35-42, 2009.

29. Xiao Q, Hu Y, Liu Y, Wang Z, Geng H, Hu L, Xu D, Wang K, Zheng L, Zheng S, et al: BEX1 promotes imatinib-induced apoptosis by binding to and antagonizing BCL-2. PLoS One 9: e91782, 2014.

30. Che ZM, Jung TH, Choi JH, Yoon DJ, Jeong HJ, Lee EJ and Kim J: Collagen-based co-culture for invasive study on cancer cells-fibroblasts interaction. Biochem Biophys Res Commun 346: 268-275, 2006

31. Gaggioli C, Hooper S, Hidalgo-Carcedo C, Grosse R, Marshall JF, Harrington K and Sahai E: Fibroblast-led collective invasion of carcinoma cells with differing roles for RhoGTPases in leading and following cells. Nat Cell Biol 9: 1392-1400, 2007.

32. Tane S, Maniwa Y, Hokka D, Tauchi S, Nishio W, Okita Y and Yoshimura M: The role of Necl-5 in the invasive activity of lung adenocarcinoma. Exp Mol Pathol 94: 330-335, 2013.

33. Friedl $\mathrm{P}$ and Wolf $\mathrm{K}$ : Tumour-cell invasion and migration: Diversity and escape mechanisms. Nat Rev Cancer 3: 362-374, 2003.

34. Wolf K, Mazo I, Leung H, Engelke K, von Andrian UH, Deryugina EI, Strongin AY, Bröcker EB and Friedl P: Compensation mechanism in tumor cell migration: Mesenchymal-amoeboid transition after blocking of pericellular proteolysis. J Cell Biol 160: 267-277, 2003.

35. Friedl P and Alexander S: Cancer invasion and the microenvironment: Plasticity and reciprocity. Cell 147: 992-1009, 2011. 